\title{
Partition of the contact force network obtained in discrete element simulations of element tests
}

\author{
Xin Huang ${ }^{1,4}$ - Catherine O’Sullivan ${ }^{2}$ - Kevin J. Hanley ${ }^{3}$ • Chung-Yee Kwok ${ }^{4}$
}

Received: 4 August 2015 / Revised: 20 November 2015 / Accepted: 14 December 2015 / Published online: 7 January 2016

(C) The Author(s) 2016. This article is published with open access at Springerlink.com

\begin{abstract}
The transmission of stress within a granular material composed of rigid spheres is explored using the discrete element method. The contribution of contacts to both deviatoric stress and structural anisotropy is investigated. The influences of five factors are considered: inter-particle friction coefficient, loading regime, packing density, contact model, and boundary conditions. The data generated indicate that using the above-average normal contact force criterion to decompose the contact force network into two subsets with distinct contributions to stress transmission and structural anisotropy is not robust. The characteristic normal contact forces marking the transition from negative to positive contribution to the overall deviatoric stress and structural anisotropy are not unique values but vary during shearing. Once the critical state is attained (i.e., once shearing continues at a constant deviator stress and solid fraction), the characteristic normal contact force remains approximately constant and this critical state characteristic normal force is observed to decrease with increasing inter-particle friction. The characteristic normal contact force considering the contribution to deviatoric stress has a power-
\end{abstract}

Catherine O'Sullivan

cath.osullivan@imperial.ac.uk

1 Department of Geotechnical Engineering, Tongji University, 1239 Siping Road, Shanghai 200092, China

2 Skempton Building, Department of Civil and Environmental Engineering, Imperial College London, London SW7 2AZ, UK

3 School of Engineering, Institute for Infrastructure and Environment, The University of Edinburgh, Edinburgh EH9 3JL, UK

4 Haking Wong Building, Department of Civil and Environmental Engineering, The University of Hong Kong, Pokfulam Road, Hong Kong, China law relationship with the mean effective stress at the critical state.

Keywords Discrete element method · Force network · Element tests $\cdot$ Stress tensor $\cdot$ Fabric tensor

\section{Introduction}

Forces in granular materials are transmitted through interparticle contacts. Physical experiments and numerical studies have revealed that the contact force network is highly heterogeneous [1-3]. Under non-isotropic stress conditions, the contact force network exhibits strong geometrical and mechanical anisotropy [4-6]. The heterogeneity of the contact force network underlies the complexity of the mechanical behavior of granular assemblies. Therefore, characterization of the contact force network is of crucial importance to understand the behavior of granular media. Radjai et al. [4] proposed that the contacts carrying above-average normal forces and the contacts bearing below-average normal forces have distinct roles in force transmission and work dissipation. Contacts bearing above-average normal forces form a 'strong' force network which transmits the majority of the applied loads. The remaining contacts form a 'weak' contact force network which stabilizes the 'strong' force network and accounts for most of the frictional energy dissipation in the system. These two sub-networks, which collectively comprise the entire contact force network, have different statistical distribution features [2]. This approach to partitioning the contact force network based on average normal force has been adopted in a number of subsequent research studies [5,7-10]. Despite its widespread adoption, there is evidence to suggest that partitioning the contact force network in this manner may not be justified. Peters et al. [11] showed that 
only approximately half of the 'strong' contacts belong to the force transmission chains, and Huang et al. [12] found that the contribution of the 'strong' contacts to frictional dissipation is considerable when inter-particle friction is high. These findings indicate that the use of the above-average criterion to partition contact force networks requires further examination.

This study uses three-dimensional discrete element modeling [13] to study the force transmission mechanism within a granular medium. The contact force characteristics are investigated following the approach of Radjai et al. [4] considering the contribution to deviatoric stress and structural anisotropy. The applicability of the above-average criterion, which was initially proposed based on the results of 2D granular simulations, to more realistic 3D scenarios is explored.

\section{DEM simulations}

Most of the simulations were run using a modified version of the LAMMPS code [14]. These three-dimensional simulations contained 20,164 idealized spherical particles. As Fig. 1a shows, the particle size distribution (PSD) of Toyoura sand was approximated with the particle diameters lying between $0.115 \mathrm{~mm}$ and $0.408 \mathrm{~mm}$ [12]. Thus the size span $\left(d_{\max }-d_{\min }\right) /\left(d_{\max }+d_{\min }\right)$ is around 0.56 , where $d_{\max }$ and $d_{\min }$ are the largest and smallest particle diameters, respectively. This is indicative of a moderate polydisperse system [3]. The samples were enclosed within a cuboidal periodic cell (Fig. 1b) and were initially isotropically compressed until the target stress state and solid fraction had been reached. They were then subjected to triaxial shearing. A simplified Hertz-Mindlin (SHM) contact model was used with a shear modulus of $29 \mathrm{GPa}$ and a Poisson's ratio of 0.12: parameters which approximate the properties of quartz sand [15]. Some periodic-cell simulations were performed using a linear elastic contact model with equal normal and tangential contact stiffnesses of $10^{5} \mathrm{~N} / \mathrm{m}$. An additional set of simulations used the same PSD and the SHM contact model, but 16,024 spheres enclosed within cylindrical rigid-wall boundaries and these simulations were run using PFC3D [16]. Gravitational body forces were neglected in all simulations. Loading was achieved by moving the upper boundary while the position of the lower boundary was fixed. A constant shear rate, $v / L_{0}$, of $1 \mathrm{~s}^{-1}$ was used for all simulations where $v$ is the velocity of moving boundary and $L_{0}$ is the corresponding initial sample vertical dimension. The inertia number was below $10^{-4}$ throughout all the simulations, indicative of quasi-static flow $[17,18]$. Three loading scenarios were applied:

- type- $a$ The sample was sheared while the lateral stresses were maintained constant;

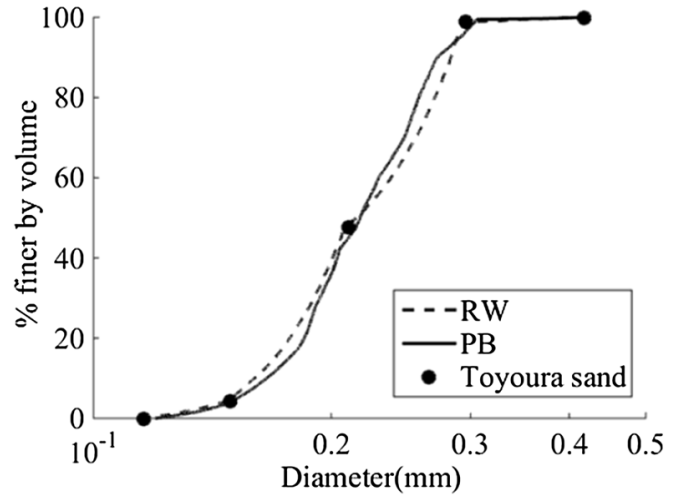

(a)

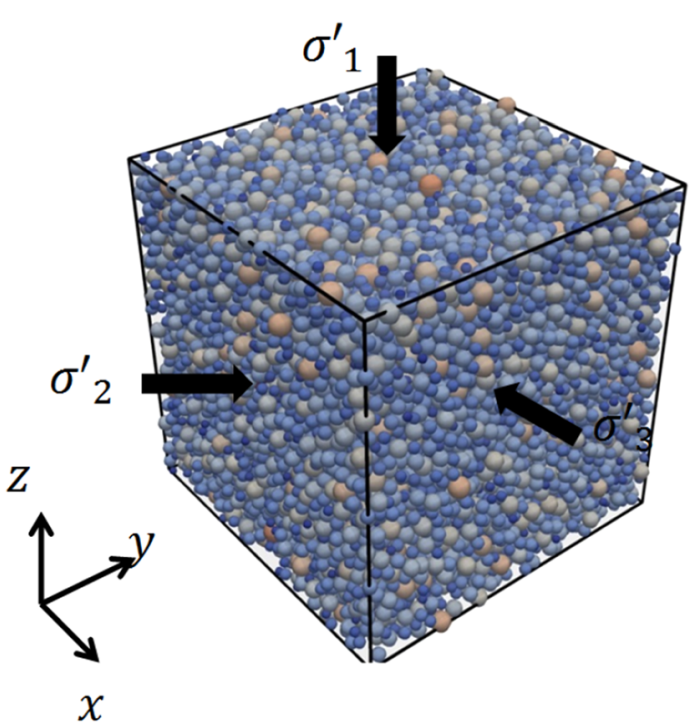

(b)

Fig. 1 Simulation setup: a particle size distribution (PB shows the particle size distribution for periodic-boundary samples; RW represents the particle size distribution for rigid-wall samples; Toyoura sand grading is obtained from sieve analysis); b loading configuration

- type- $b$ The sample was sheared with a constant-volume constraint imposed;

- type-c The sample was sheared while the positions of lateral boundaries were adjusted accordingly to keep a constant mean stress $p=\left(\sigma_{1}+\sigma_{2}+\sigma_{3}\right) / 3$ where $\sigma_{1}, \sigma_{2}$, and $\sigma_{3}$ are the major, intermediate, and minor principal stresses, respectively.

In all the three loading regimes, the lateral stresses were kept approximately identical, i.e., $\sigma_{2}=\sigma_{3}$. Therefore, the deviatoric stress can be simplified as $q=\sigma_{1}-\sigma_{3}$ and the mean stress $p$ equals to $1 / 3\left(\sigma_{1}+2 \sigma_{3}\right)$. Five different inter-particle friction coefficients $(\mu)$ were used for the type- $a$ loading scenario to investigate the influence of $\mu$ on the stress transmission characteristics, while only $\mu=0.25$ was applied to the type- $b$ and type- $c$ simulations. In all cases shearing 
continued to a sufficiently high strain level that critical state conditions were attained, i.e., the combination of stress level and packing density beyond which shearing continues at a constant deviator stress, constant mean stress, and constant packing density $[19,20]$.

\section{Results and discussion}

The stress and deformation responses of a representative subset of the simulations subject to the type- $a$ loading condition using periodic boundaries and the SHM contact model are illustrated in Fig. 2. All these simulations considered the same initial isotropic conditions prior to shearing (initial solid fraction, $s$, of 0.652 and an initial isotropic mean stress, $p_{0}$, of $100 \mathrm{kPa}$ ) and the confining pressure was maintained at 100 $\mathrm{kPa}$ during shearing. Referring to Fig. 2a, for all samples the deviatoric stress $q$ initially increases rapidly to a peak value and subsequently decreases to become almost constant when the axial strain $\varepsilon_{a}$ exceeds $20 \%$. The deviatoric stress initially increases with increasing $\mu$ but becomes insensitive to

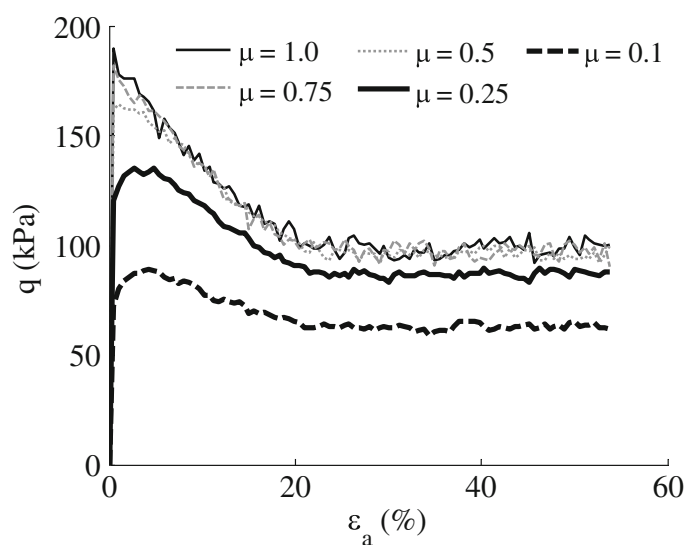

(a)

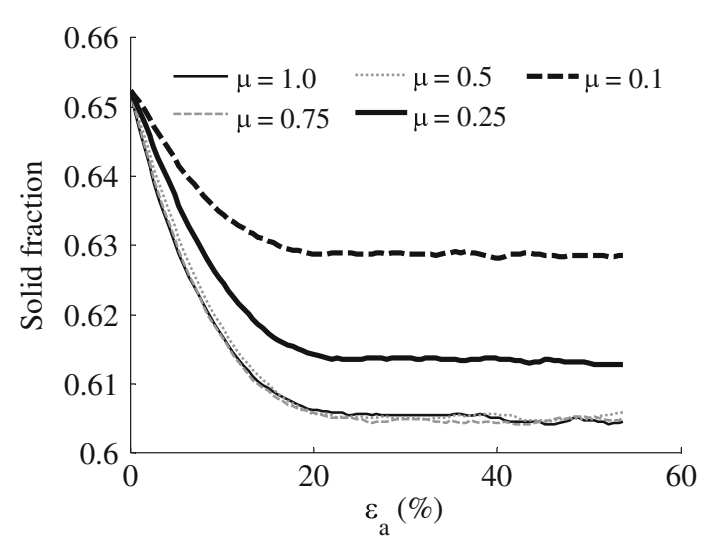

(b)

Fig. 2 Effect of $\mu$ on the evolution of $q$ (a) and solid fraction (b) during simulations using periodic boundaries and the type- $a$ loading protocol $\mu$ when $\mu$ exceeds 0.5 due to the interplay between rotational behavior and sliding behavior at the contacts [12]. Figure $2 b$ shows that all samples dilate from their initially high density and attain nearly constant solid fractions that decrease with increasing $\mu$. The constant deviatoric stress and solid fraction at large $\varepsilon_{a}$ levels represent the typical critical state (steady-state) response which is the ultimate state that a soil can approach $[19,20]$.

The average stress within a granular assembly can be calculated directly from the contact forces [21]

$\sigma_{i j}=\frac{1}{V} \sum_{1}^{N_{\mathrm{c}}} f_{i} l_{j}$

in which $V$ is the volume of the domain considered, $f_{i}$ is the $i$ th component of the contact force vector $f, l_{j}$ is the $j$ th component of the branch vector $\boldsymbol{l}$ that joins the centers of two touching particles and $N_{c}$ is the number of contacts. The particle velocities and particle inertias are sufficiently small that the dynamic component of the stress tensor (as described in Claudin [22]) can be neglected. Following the approach of Radjai et al. [4], Fig. 3 presents the cumulative contribution of contact forces to the overall deviatoric stress as a function of $f_{n} /\left\langle f_{n}\right\rangle$ for the type- $a$ simulations considered in Fig. 2 at $\varepsilon_{a}=30 \%$ (at this axial strain level the critical state has been attained in all the simulations); $\left\langle f_{n}\right\rangle$ is the mean normal contact force over the entire assembly and the corresponding $q$ value for each $f_{n} /\left\langle f_{n}\right\rangle$ considers the contribution of the subset of contacts with $f_{n} /\left\langle f_{n}\right\rangle$ below the current value. To create Fig. 3, the contacts are firstly sorted in an ascending order according to the magnitude of $f_{n} /\left\langle f_{n}\right\rangle$ (i.e., the $\xi$ parameter in Radjai et al. [4]). The contribution of each contact to $q$ is then calculated based on Eq. 1. Each data point in Fig. 3 represents the cumulative sum of the

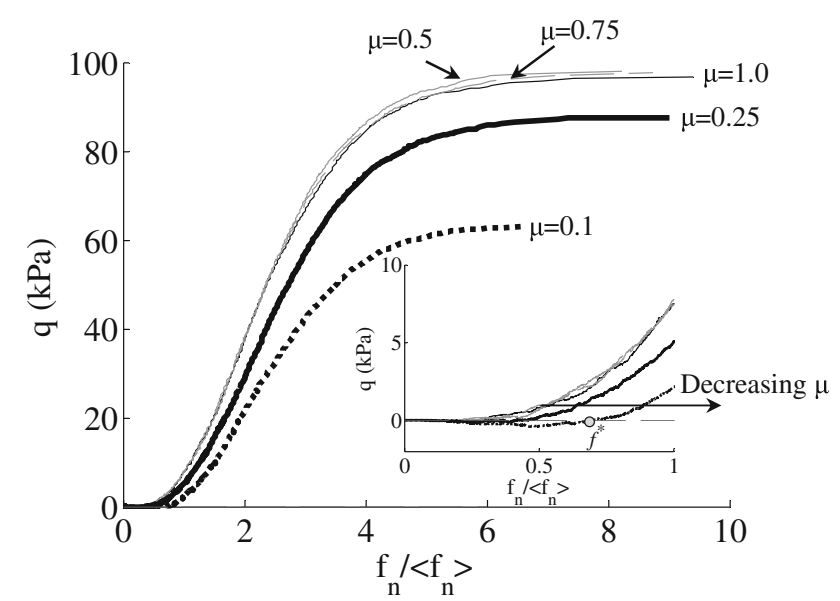

Fig. 3 Accumulated $q$ as a function of $f_{n} /\left\langle f_{n}\right\rangle$ at $30 \%$ axial strain (type-a loading, $s=0.652, p_{0}=100 \mathrm{kPa}$ ) 

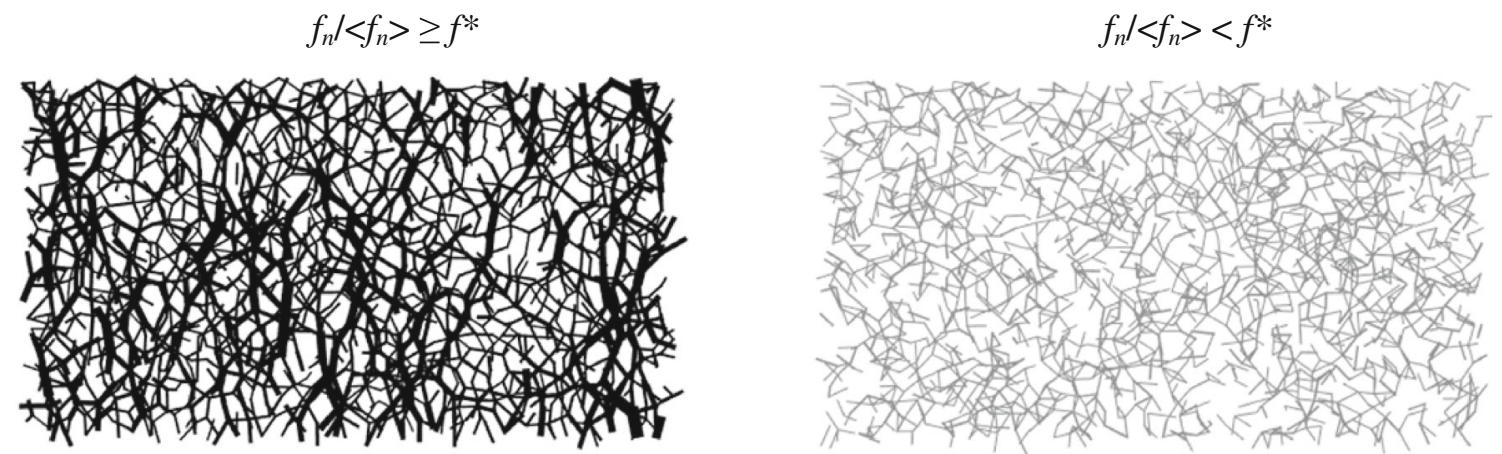

(a) $\mu=0.1$
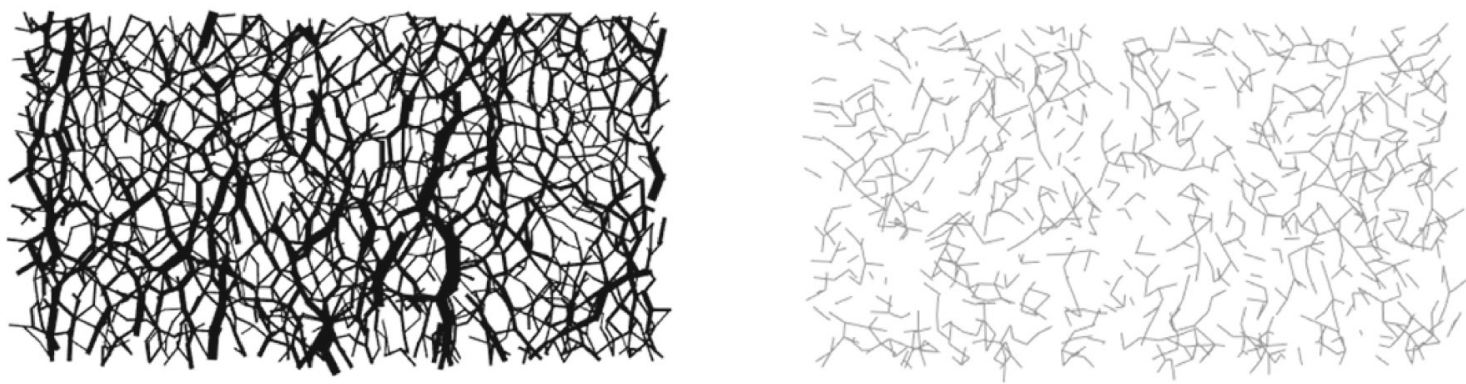

(b) $\mu=0.5$
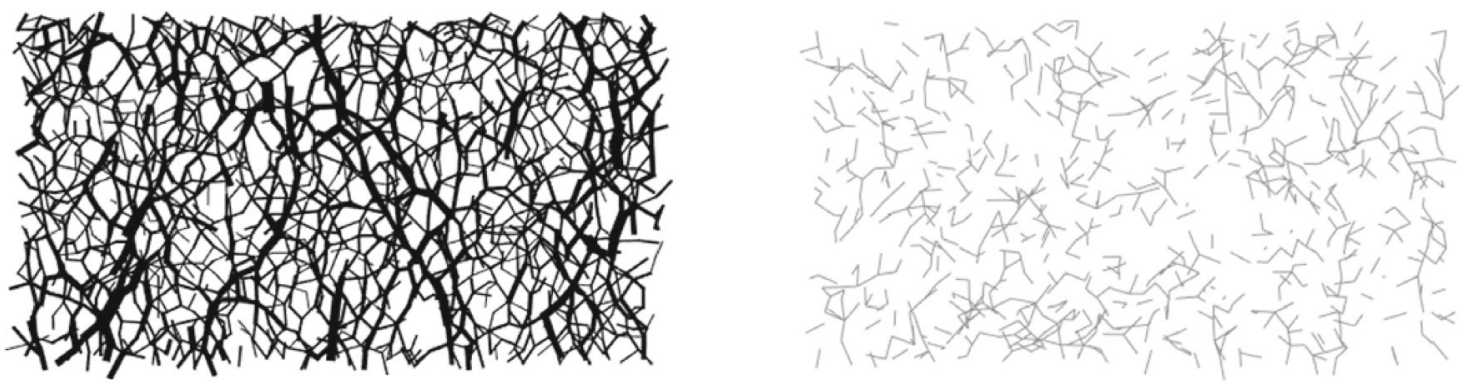

(c) $\mu=1.0$

Fig. 4 Projection of normal contact force on the $\mathrm{X}\left(\sigma_{3}\right)-\mathrm{Z}\left(\sigma_{1}\right)$ plane at the critical state $\left(\varepsilon_{a}=30 \%\right)$

contributions to $q$ considering contacts with $f_{n} /\left\langle f_{n}\right\rangle$ below the current value. There is a range of $f_{n} /\left\langle f_{n}\right\rangle$ values where the cumulative curves give $q<0$. When the stress tensor is calculated considering only contacts with force values lying in this range, the resultant principal stress orientation will differ from the overall principal stress orientation by more than $45^{\circ}$. An enlarged view of the range $0<f_{n} /\left\langle f_{n}\right\rangle<1$ is superimposed on the figure to enable a closer examination of the transition from negative to positive contributions to $q$. Contacts that make a positive contribution to $q$ are on average considered part of the force transmission network following Radjai et al. [4]. The data show that at the strain level considered the characteristic normal contact force $f^{*}$ which separates the positive and negative contributions to $q$ is smaller than unity for all the $\mu$ values considered.

Figure 4 presents the projection of the normal contact force onto the $\mathrm{X}\left(\sigma_{3}\right)-\mathrm{Z}\left(\sigma_{1}\right)$ plane at $\varepsilon_{a}=30 \%$.
For clarity, only contacts within the middle part of the samples (10\% of the entire sample) are considered. The contacts are represented by lines connecting the centers of the particles in contact, and the thickness of each line is proportional to the magnitude of $f_{n} /\left\langle f_{n}\right\rangle$. The overall contact force network is divided into two subsets according to the magnitude of $f_{n} /\left\langle f_{n}\right\rangle$ with respect to $f^{*}$, i.e., $f_{n} /\left\langle f_{n}\right\rangle \geq f^{*}$ (on the left in black) and $f_{n} /\left\langle f_{n}\right\rangle<f^{*}$ (on the right in gray). For the $\mu$ values considered, the configurations of contact force networks are quite similar. The contacts within the $f_{n} /\left\langle f_{n}\right\rangle \geq f^{*}$ subset are oriented along the major principal stress direction, while the orientations of the contacts belonging to the $f_{n} /\left\langle f_{n}\right\rangle<f^{*}$ have a random orientation. This reflects the distinct roles of the two subsets: the $f_{n} /\left\langle f_{n}\right\rangle \geq f^{*}$ subset acts as force-bearing backbones carrying the majority of deviatoric loading, while the $f_{n} /\left\langle f_{n}\right\rangle<f^{*}$ subset is the supporting 


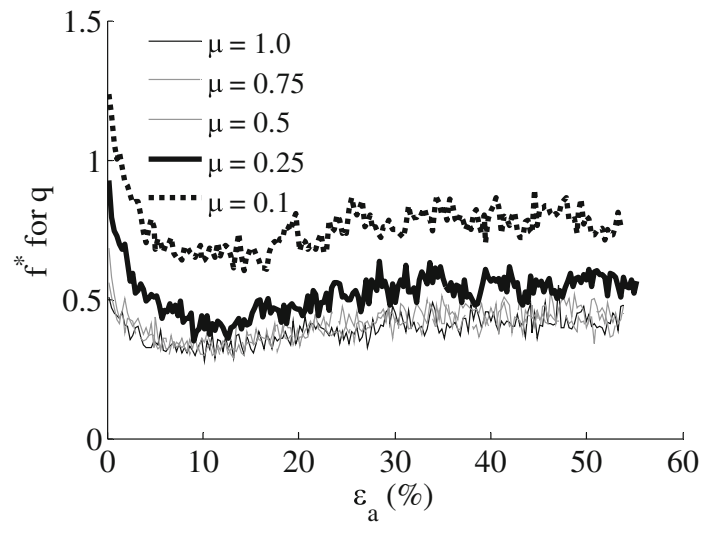

Fig. 5 Influence of $\mu$ on the evolution of $f^{*}$ considering the contribution to $q$ (type- $a$ loading, $s=0.652$ and $p_{0}=100 \mathrm{kPa}$ )



(a)

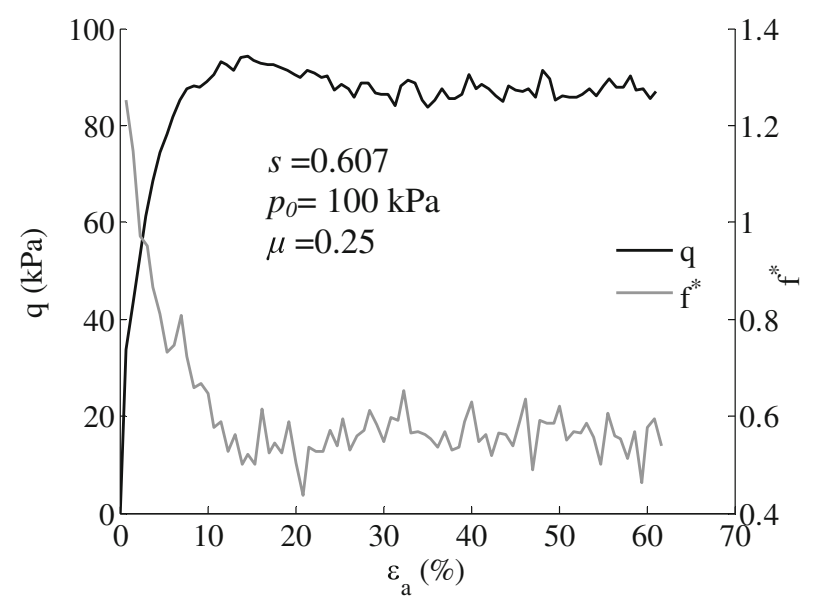

(c)

Fig. 6 Evolution of $f^{*}$ considering the contribution to $q$ with axial strain: a for a representative simulation using the type- $b$ loading proto$\mathrm{col} ; \mathbf{b}$ for a representative simulation using the type-c loading protocol; network which provides lateral support to the force-bearing network.

Figure 5 shows the evolution of $f^{*}$ for $q$ during shearing for the simulations presented in Fig. 2. For all the $\mu$ values considered, $f^{*}$ for $q$ is relatively high at the isotropic state and varies during subsequent shearing, tending to nearly a constant value at the critical state $\left(\varepsilon_{a} \geq 30 \%\right)$. Moreover, at all strain levels $f^{*}$ increases with decreasing $\mu$. The structural anisotropy also increases as $\mu$ increases [12]. The non-uniqueness of $f^{*}$ for $q$ is also evident for the type- $b$ (Fig. 6a) and the type-c (Fig. 6b) loading scenarios, for the type- $a$ loading using the linear elastic model (Fig. $6 c)$ and for the type- $a$ loading using the rigid-wall boundaries (Fig. 6d). Figures 3, 4, 5, and 6 indicate that many of the contacts with $0<f_{n} /\left\langle f_{n}\right\rangle<1$ which have been

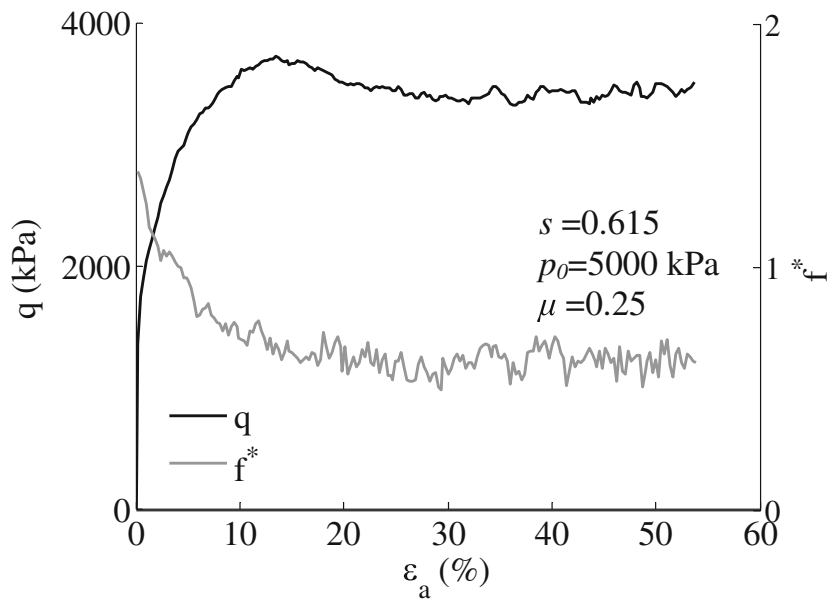

(b)

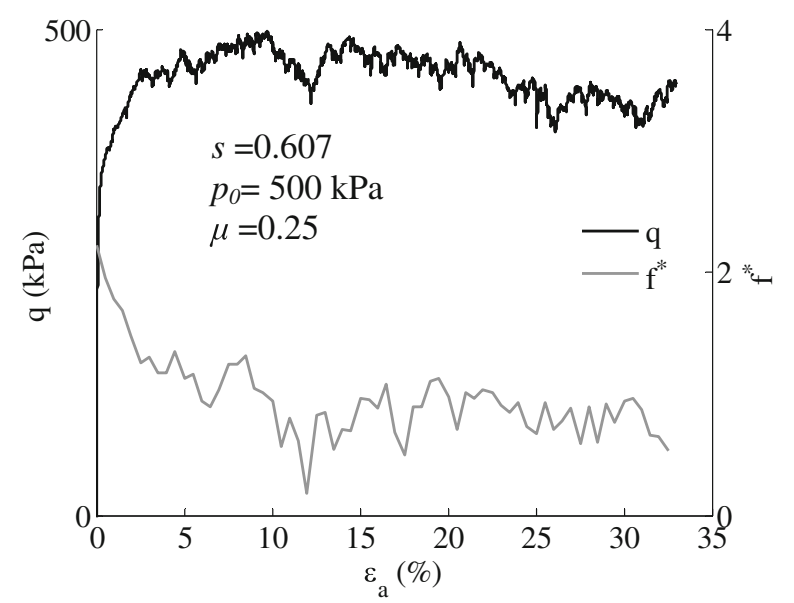

(d)

c for a representative simulation using the type- $a$ loading protocol and the linear elastic contact model; $\mathbf{d}$ for a representative simulation using rigid-wall boundaries and the type- $a$ loading protocol 
hitherto considered 'weak' are not only contributing to the secondary supporting force network but also to the deviatoric load and so their classification as "weak" may not be appropriate.

The contact anisotropy is usually quantified using the fabric tensor [23]:

$\Phi_{i j}=\frac{1}{N_{\mathrm{c}}} \sum_{k=1}^{N_{\mathrm{c}}} n_{i}^{k} n_{j}^{k}$

where $n_{i}^{k}$ denotes the unit contact normal. The degree of contact anisotropy is evaluated using deviatoric fabric defined here as $\Phi_{d}=\sqrt{\frac{1}{2}\left[\left(\Phi_{1}-\Phi_{2}\right)^{2}+\left(\Phi_{1}-\Phi_{3}\right)^{2}+\left(\Phi_{2}-\Phi_{3}\right)^{2}\right]}$, where $\Phi_{1}, \Phi_{2}$, and $\Phi_{3}$ are the major, intermediate, and minor principal fabrics, respectively, and these are the eigenvalues for the fabric tensor. It was found that $\Phi_{2}$ and $\Phi_{3}$ are effectively identical in the simulations as a $\sigma_{2}=\sigma_{3}$ condition was imposed. Therefore, the deviatoric fabric can be simplified as $\Phi_{d}=\Phi_{1}-\Phi_{3}$. Figure 7 shows $\Phi_{d}$ as a function of $f_{n} /\left\langle f_{n}\right\rangle$ at the critical state for each $\mu$ value considered. To create Fig. 7, the contacts are firstly sorted in an ascending order according to the magnitude of $f_{n} /\left\langle f_{n}\right\rangle$, as was required for Fig. 3. The contribution of each contact to $\Phi_{d}$ is then calculated based on Eq. 2. Each data point in Fig. 7 represents the cumulative sum of the contribution to $\Phi_{d}$ considering the contacts with $f_{n} /\left\langle f_{n}\right\rangle$ below the current value. Analogous to the stress calculations described above, there is a range of $f_{n} /\left\langle f_{n}\right\rangle$ values that give $\Phi_{d}<0$ on the cumulative curves, and the fabric tensor calculated considering only contacts with force values lying in this range gives a principal fabric orientation that differs from the overall principal fabric orientation by more than $45^{\circ}$. It can be seen from Fig. 7 that $\Phi_{d}$ increases as $\mu$ increases. This is because with increasing $\mu$, the inherent stability of the force-bearing skeleton increases,

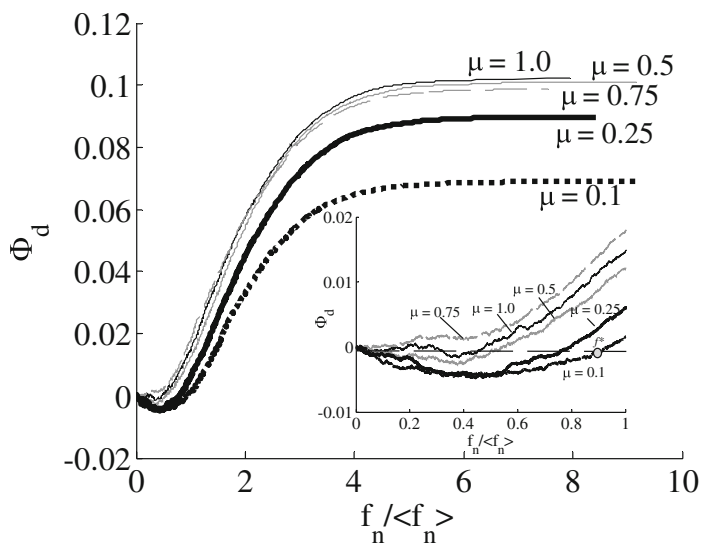

Fig. 7 Accumulated $\Phi_{d}$ as a function of $f_{n} /\left\langle f_{n}\right\rangle$ at the critical state (type- $a$ loading, $s=0.652, p_{0}=100 \mathrm{kPa}$ )

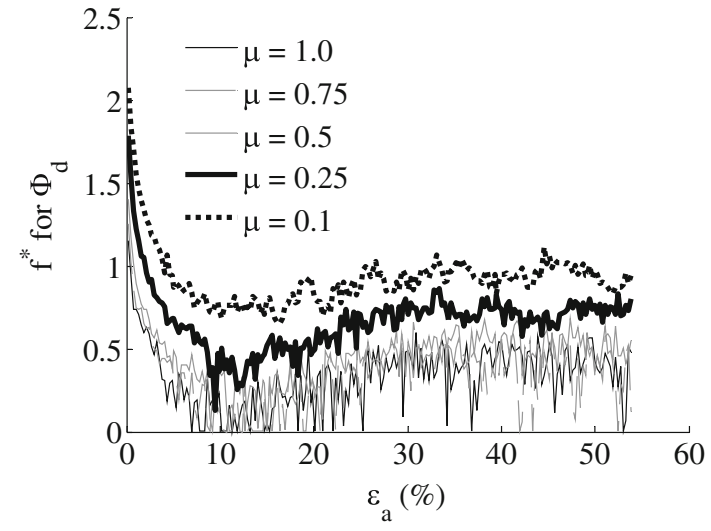

Fig. 8 Influence of $\mu$ on the evolution of $f^{*}$ considering the contribution to $\Phi_{d}$ (type- $a$ loading, $s=0.652$ and $p_{0}=100 \mathrm{kPa}$ )

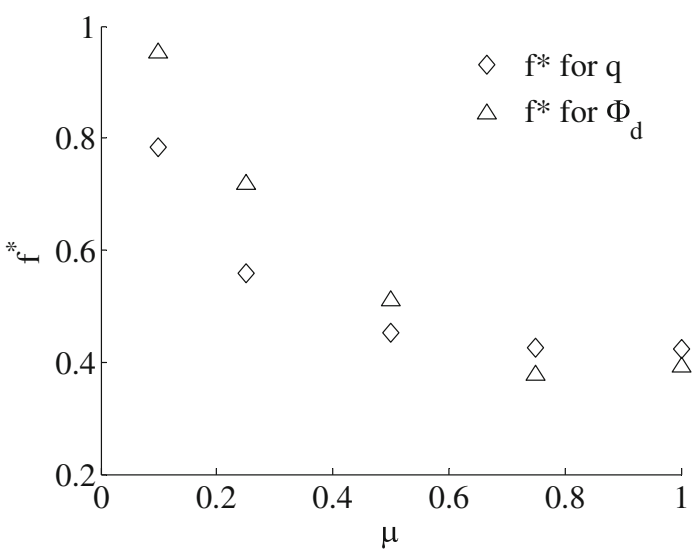

Fig. 9 Variation of $f^{*}$ at the critical state with $\mu$ for representative simulations using the type- $a$ loading protocol

the lateral supporting contacts orthogonal to the strong force chains are not activated, and hence the anisotropy increases. The inset in Fig. 7 shows that for all $\mu$ values considered, the $f^{*}$ value which marks the transition from a negative contribution to a positive contribution to $\Phi_{d}$ generally decreases with increasing $\mu$ value. Figure 8 presents the variation of $f^{*}$ considering the contribution to $\Phi_{d}$ with axial strain $\left(\varepsilon_{a}\right)$ during shearing for a representative set of the type- $a$ simulations. It is clear that just as $f^{*}$ for $q$ varied, $f^{*}$ for $\Phi_{d}$ is also not a constant; it depends on $\mu$ and the strain level. Similar to $f^{*}$ for $q, f^{*}$ for $\Phi_{d}$ also remains approximately constant when the axial strain $\varepsilon_{a}$ exceeds $30 \%$ and critical state shearing conditions are attained. Figure 9 illustrates the variation of $f^{*}$ at the critical state with $\mu$ considering both contributions to $q$ and $\Phi_{d} . f^{*}$ values defined for $q$ differ from those defined for $\Phi_{d}$; the former are smaller at small $\mu$ values $(<0.75)$ but the two become almost identical at $\mu$ values $\geq 0.75$. Similar observations can also be made for other types of loading conditions for which data are not shown herein for conciseness. This is because apart from geometrical anisotropy $\Phi_{d}, q$ is 


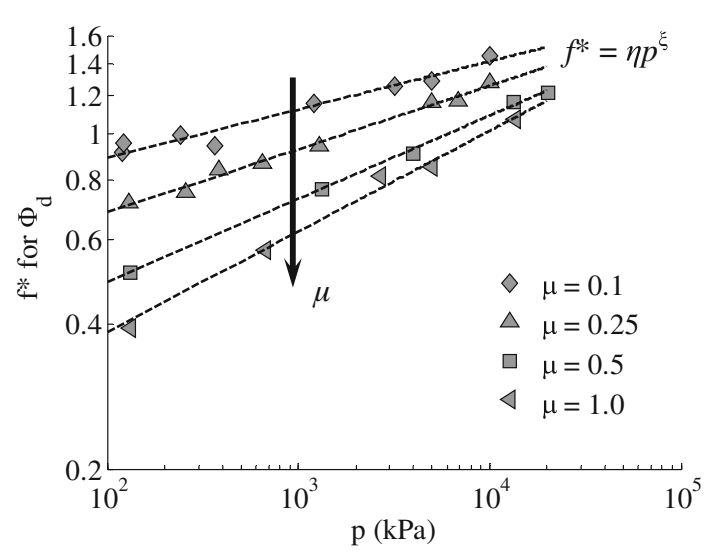

(a)

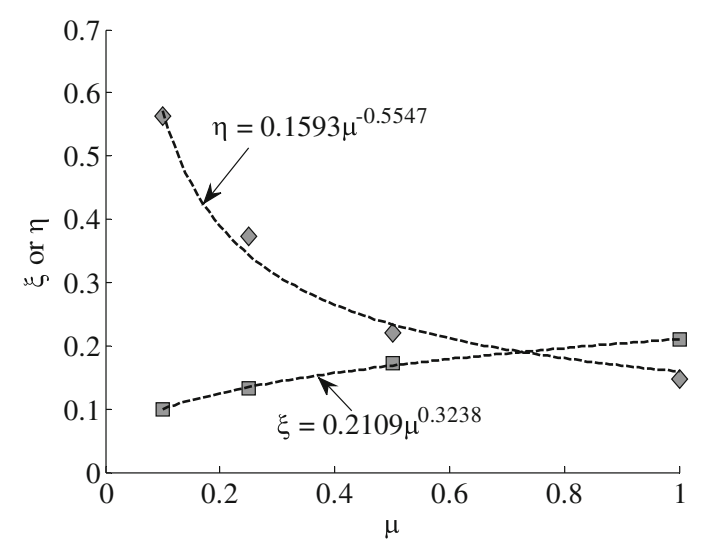

(b)

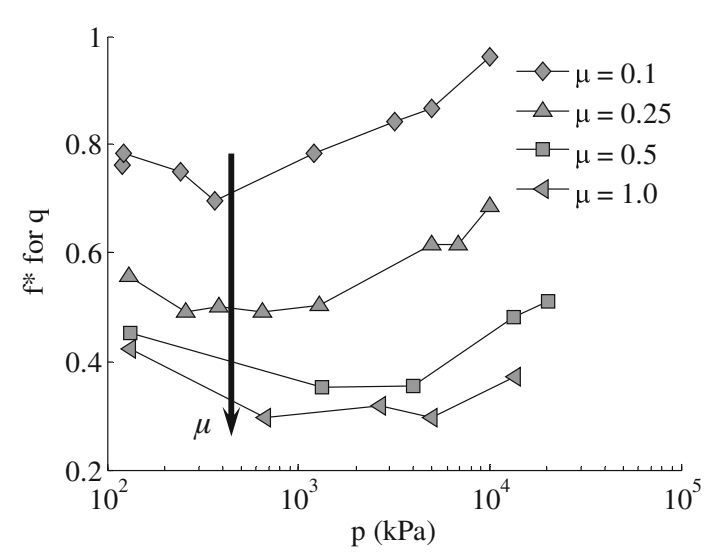

(c)

Fig. 10 Correlations between the characteristic force $f^{*}$ with mean stress $p$ at the critical state: a critical state $f^{*}$ for $\Phi_{d}$ with $p$; b variation of power-law regression parameters with $\mu$; c critical state $f^{*}$ for $q$ with $p$

also a function of mechanical anisotropies $[4,24]$. Both $f^{*}$ for $q$ and $f^{*}$ for $\Phi_{d}$ decrease with increasing $\mu$.

As shown in Figures 5, 6, and 8, just as is the case for the stress and volumetric responses at large strain levels, both $f^{*}$ for $q$ and $f^{*}$ for $\Phi_{d}$ become almost constant at the critical
Table 1 Regression parameters for the relationship between $f^{*}$ for $\Phi_{d}$ and $p$ at the critical state

\begin{tabular}{lllll}
\hline & $\mu=0.1$ & $\mu=0.25$ & $\mu=0.5$ & $\mu=1.0$ \\
\hline$\eta$ & 0.5622 & 0.3731 & 0.2207 & 0.1475 \\
$\xi$ & 0.09997 & 0.1318 & 0.1732 & 0.2090 \\
\hline
\end{tabular}

state. Figure 10a shows the relationship between the critical state $f^{*}$ for $\Phi_{d}$ and the mean stress $p$ at the critical state. Due to the presence of oscillations, the critical state data presented in Fig. 10a are taken as the mean values of the corresponding quantities for the last $10 \%$ of axial strain. $f^{*}$ for $\Phi_{d}$ at the critical state increases as $p$ increases but decreases with increasing $\mu$, in line with Figs. 7 and 8. This is the opposite of the correlations between $\Phi_{d}$ at the critical state and $p^{\prime}$ and between $\Phi_{d}$ and $\mu$ noted in [12]. The relationships between $f^{*}$ for $\Phi_{d}$ for different $\mu$ values can be represented by a series of power-law functions, i.e., in a form of $f^{*}=\eta p^{\xi}$. The fitting parameters $\eta$ and $\xi$ for each $\mu$ case are given in Table 1. $\eta$ decreases with increasing $\mu$ while the opposite trend is evident for $\xi$. Figure $10 \mathrm{~b}$ presents the variation of $\eta$ and $\xi$ with $\mu$. Both of them follow a power-law relationship with $\mu$. $\eta$ decreases with increasing $\mu$ and thus the fitting function has a negative power, while $\xi$ increases with increasing $\mu$ and the fitting function has a positive power. Figure 10c plots $f^{*}$ for $q$ at the critical state against $p$. In comparison to $f^{*}$ for $\Phi_{d}$, the relationship between the critical state $f^{*}$ for $q$ and $p$ is more complicated; for all the $\mu$ values considered, it initially decreases with increasing $p$, reaches a local minimum and then continues to increase as $p$ is further increased.

\section{Conclusions}

This study presents a systematic investigation on the force transmission characteristics within granular media. The influences of multiple factors were considered including inter-particle friction coefficient, loading regime, packing density, contact model, and boundary conditions. The evidence presented in this study clearly suggests that some of the weak contacts carrying below-average normal contact forces also participate in stress transmission and contribute to structural anisotropy. It is shown that both the characteristic normal contact force which marks the transition from a negative to a positive contribution to the overall deviatoric stress and that marking the transition from a negative to a positive contribution to structural anisotropy vary until the critical state is reached and remain approximately constant thereafter. The characteristic normal contact force for the deviatoric stress and that for the structural anisotropy are not unique and differ from each other. Thus, using the average normal contact force $\left\langle f_{n}\right\rangle$ as the transition point to sepa- 
rate a load-bearing network which is anisotropic and aligned along the external loading direction from a supporting network which is isotropic and orthogonal to the load-bearing network within a granular packing is not robust for threedimensional systems.

Open Access This article is distributed under the terms of the Creative Commons Attribution 4.0 International License (http://creativecomm ons.org/licenses/by/4.0/), which permits unrestricted use, distribution, and reproduction in any medium, provided you give appropriate credit to the original author(s) and the source, provide a link to the Creative Commons license, and indicate if changes were made.

\section{References}

1. Majmudar TS, Behringer R (2005) Contact force measurements and stress-induced anisotropy in granular materials. Nature 435:1079-1082

2. Radjai F, Jean M, Moreau J, Roux S (1996) Force distributions in dense two-dimensional granular systems. Phys Rev Lett 77:274277

3. Voivret C, Radjaï F, Delenne J-Y, El Youssoufi M (2009) Multiscale force networks in highly polydisperse granular media. Phys Rev Lett 102:178001

4. Radjai F, Wolf DE, Jean M, Moreau J (1998) Bimodal character of stress transmission in granular packings. Phys Rev Lett 80:61-64

5. Guo N, Zhao J (2013) The signature of shear-induced anisotropy in granular media. Comput Geotech 47:1-15

6. Bathurst RJ, Rothenburg L (1992) Micromechanical features of granular assemblies with planar elliptical particles. Géotechnique 42:79-95

7. Antony S, Kruyt N (2009) Role of interparticle friction and particle-scale elasticity in the shear-strength mechanism of threedimensional granular media. Phys Rev E 79:031308

8. Tordesillas A (2007) Force chain buckling, unjamming transitions and shear banding in dense granular assemblies. Philos Mag $87: 4987-5016$
9. Ben-Nun O, Einav I, Tordesillas A (2010) Force attractor in confined comminution of granular materials. Phys Rev Lett 104:108001

10. Thornton C, Antony SJ (2000) Quasi-static shear deformation of a soft particle system. Powder Technol 109:179-191

11. Peters JF, Muthuswamy M, Wibowo J, Tordesillas A (2005) Characterization of force chains in granular material. Phys Rev E 72:1-8

12. Huang X, Hanley K, O'Sullivan C, Kwok CY (2014) Exploring the influence of inter-particle friction on critical state behaviour using DEM. Int J Numer Anal Methods Geomech 38:1276-1297

13. Cundall PA, Strack ODL (1979) A discrete numerical model for granular assemblies. Géotechnique 29:47-65

14. Plimpton S (1995) Fast parallel algorithms for short-range molecular dynamics. J Comput Phys 117:1-19

15. Simmons G, Brace WF (1965) Comparison of static and dynamic measurements of compressibility of rocks. J Geophys Res 70:5649-5656

16. Itasca Consulting Group (2007) PFC3D Version 4.0 User Manual. Itasca Consulting Group, Minneapolis

17. Peyneau P, Roux J-N (2008) Frictionless bead packs have macroscopic friction, but no dilatancy. Phys Rev E 78:011307

18. Da Cruz F, Emam S, Prochnow M, Roux J-N, Chevoir F (2005) Rheophysics of dense granular materials: Discrete simulation of plane shear flows. Phys Rev E 72:021309

19. Schofield AN, Wroth CP (1958) On the yielding of soils. Géotechnique 8:22-53

20. Schofield A, Wroth P (1968) Critical state soil mechanics. McGraw-Hill, London

21. Bagi K (1996) Stress and strain in granular assemblies. Mech Mater 22:165-177

22. Claudin $P$ (2007) Chapter 14 static properties of granular materials in granular physics. Cambridge University Press, Cambridge, pp 223-273

23. Satake M (1982) Fabric tensor in granular materials. In: Vermeer PA, Luger HJ (eds) IUTAM symposium: deformation and failure of granular materials, Balkema, Rotterdam, pp 63-68

24. Rothenburg L, Bathurst RJ (1989) Analytical study of induced anisotropy in idealized granular material. Géotechnique 39:601614 